\title{
Speckle reduction system by using spatial and temporal scrambler in integrating sphere
}

Michel Sebastien, F. L. Carbo, W. Glastre, S. Gaillac, M. Cortese, et al.

Michel Sebastien, F. L. Carbo, W. Glastre, S. Gaillac, M. Cortese, E. Compain, "Speckle reduction system by using spatial and temporal scrambler in integrating sphere," Proc. SPIE 11852, International Conference on Space Optics - ICSO 2020, 1185236 (11 June 2021); doi: 10.1117/12.2599534

SPIE Event: International Conference on Space Optics - ICSO 2021, 2021, Online Only 


\section{International Conference on Space Optics-ICSO 2020}

Virtual Conference

30 March-2 April 2021

Edited by Bruno Cugny, Zoran Sodnik, and Nikos Karafolas
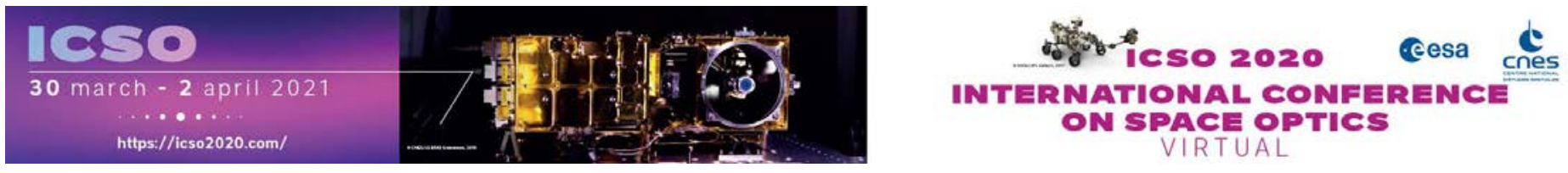

\section{Speckle reduction system by using spatial and temporal scrambler in integrating sphere}

\section{Cesa isoporecestings denes}




\title{
Speckle reduction system by using spatial and temporal scrambler in integrating sphere
}

\author{
S. Michel*a, F.L. Carbo ${ }^{b}$, W. Glastre ${ }^{a}$, S. Gaillac ${ }^{a}$, M.Cortese $^{b}$, E. Compain ${ }^{a}$, \\ ${ }^{a} B e r t i n$ Technologies, 155 rue Louis Armand, les Milles, 13290, Aix-en-Provence, France \\ ${ }^{\mathrm{b} O H B}$ System AG, Manfred-Fuchs-Str. 1, D-82234 Weßling, Germany \\ *sebastien.michel@bertin.fr; phone +33(0)4 42604 646; bertin-technologies.com
}

\begin{abstract}
The OGSE GESTA (Geometrical and Spectral Test Assembly) operates in vacuum chamber to perform the optical characterization of the IRS instrument. GESTA integrates both sources, gas cell and laser sources, in order to perform a spectral characterization and calibration.

The MTF measurements are performed by measuring the signal coming from a hot pattern plate, illuminated by a stable and homogeneous optical beam. To measure the background signal, the pattern plate is removed and a cryogenic blackbody is used instead of.

This paper describes this illumination sub-system used in GESTA, developed by Bertin Technologies, and using the infrared laser sources. The principle of the used solution is to apply a dynamic variation to the interference status responsible for the speckle at the output of the integrating sphere, in order to eliminate the impact of the spatial coherence of the output beam and to get a uniform radiance through temporal averaging over the integration time of the optical detector.

For that, the laser beams hit a rotating diffusing disc located inside the sphere. For GESTA, the requirement is challenging as speckle contrasts need to be as low as $0.1 \%$ for a $400 \mu$ s integration time. The solution developed shows good enhancements, in terms of radiance level, stability, uniformity, and is easily translatable to other spectral ranges. Final achieved performances and the related influencing parameters are reported.
\end{abstract}

Keywords: Speckle, MTF measurement, Satellite, OGSE GESTA, MeteoSat Third Generation

*sebastien.michel@bertin.fr; phone +33(4) 42604646. 


\section{INTRODUCTION}

\subsection{The MTG-S mission and its IRS instrument}

Meteosat Third Generation - Infrared Sounder (MTG-IRS), manufactured by OHB Munich, will be launched in Geostationary orbit and will perform Earth observations in the near and far infrared spectrum (MWIR and LWIR range).

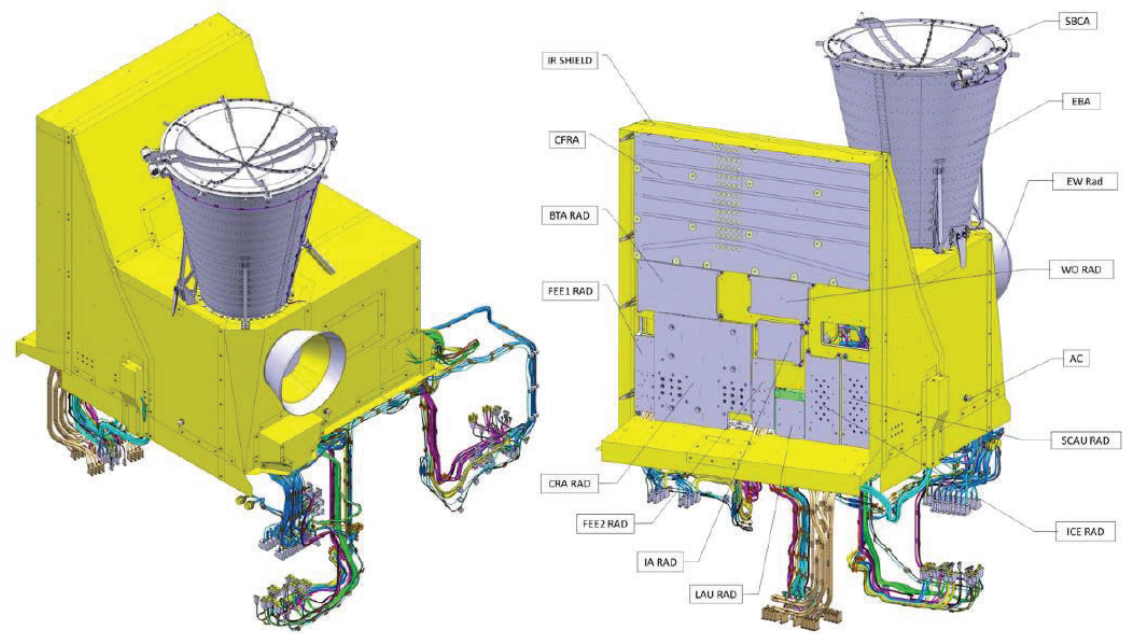

Figure 1. General view of the IRS instrument

The primary objective of the IRS mission is to support numerical weather prediction at regional and global scales, through the provision of atmospheric motion vectors with higher vertical resolution (to be extracted from the tracking of three-dimensional water vapour patterns) and more frequent information on temperature and water vapour profiles.

The secondary objective of the IRS mission, in relation with other MTG instruments, is to support chemical weather and air quality applications e.g. observation of the diurnal cycle of atmospheric trace gases, including their spatial variability, horizontal transport, and potentially vertical exchange associated with weather and climate processes.

The InfraRed Sounding (IRS) mission covers the full Earth disc, providing hyperspectral sounding information in Long Wave InfraRed (LWIR: 8.26 to $14.71 \mu \mathrm{m}$ ) and Mid Wave InfraRed (MWIR: 4.44 to $6.25 \mu \mathrm{m}$ ) band with a spatial sampling distance of nearly $4 \mathrm{~km}$.

The MTG-IRS instrument is an Imaging Fourier Transform Spectrometer mainly composed of a Michelson-type interferometer and two infrared detectors covering the MWIR and LWIR bands. The basic principle of a Michelson's interferometer is to acquire interferograms at the detectors of the instrument by varying the optical path difference between two interferometer arms for a period of roughly ten seconds (equivalent to one stare). The signal acquired by the instrument for one stare is composed of 51,200 interferograms, which upon Fourier-Transformation reveal the spectral content of the input scene. 


\subsection{GESTA OGSE Overview}

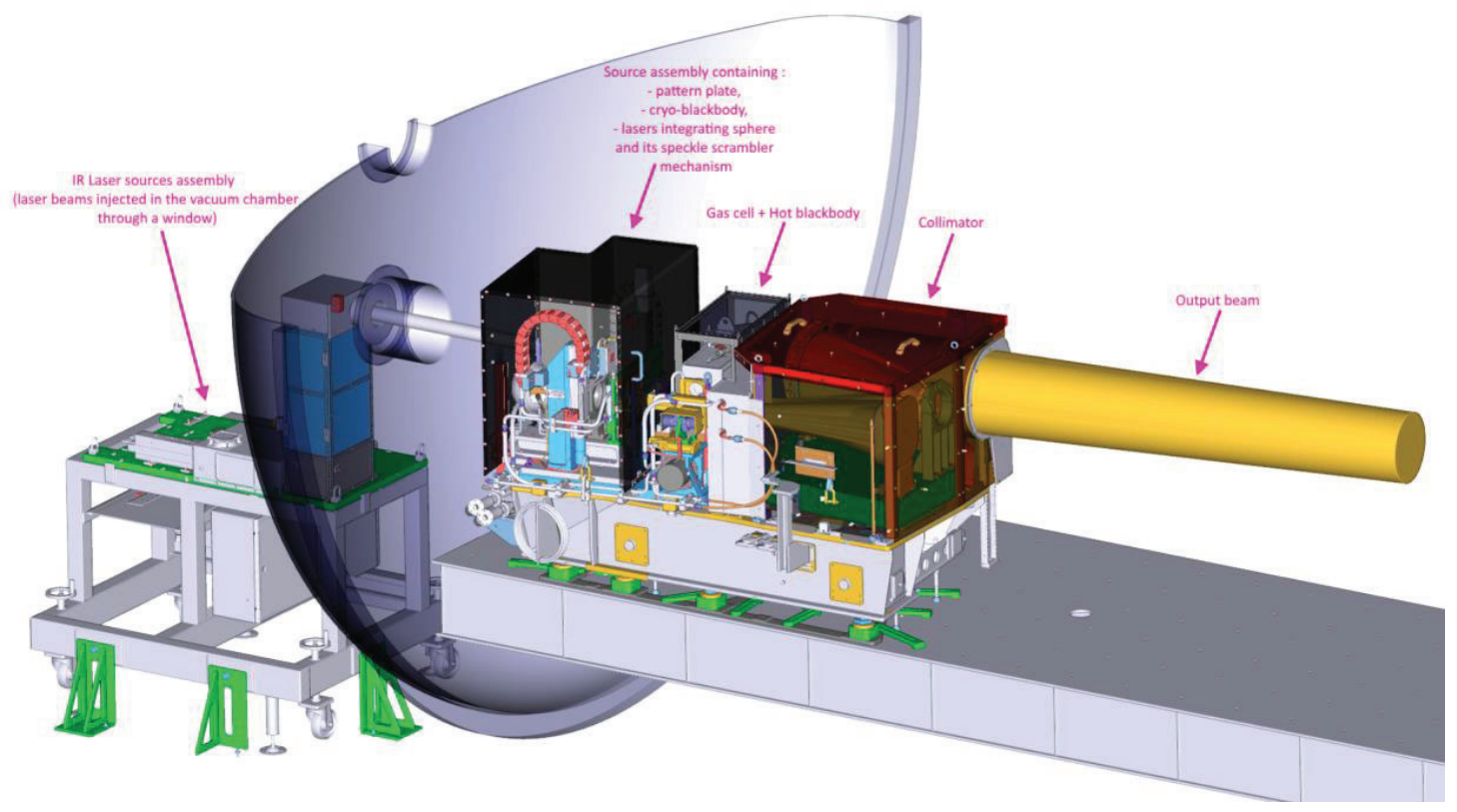

Figure 2. 3D view of the GESTA OGSE in its vacuum chamber

Before launch, the IRS instrument will undergo optical performance and calibration tests in vacuum in order to verify its geometrical, spectral, and radiometric performances. For the geometric and spectral measurements, a custom OGSE (Optical Ground Support Equipment) called GESTA (Geometric and Spectral Test Assembly) will be used. The radiometric needs will be covered by custom blackbodies OGSEs not discussed in this paper.

More specifically, the GESTA OGSE will allow to perform the following tests on the completely assembled IRS in the two IR spectral channels (MWIR and LWIR):

- Integrated Energy measurement and characterization

- Spectral characterization (Instrument Line Shape - ILS)

- Geometric characterization (spatial sampling distance (SSD) and spatial sampling error (SSE))

- Intra- and Inter-Band Co-Registration measurements

- End-to-end spectral test by using a gas cell

- Straylight measurement and characterization

For that end, the GESTA will use three different configurations (see Figure 3).

- Configuration \#1: Gas Cell configuration

- Configuration \#2: Pattern plate + integrating sphere + IR lasers

- Configuration \#3: Pattern plate + Cold blackbody

This paper will mainly focus on configuration \#2 and the lasers speckle scrambling mechanism used to reach sufficient spatial and temporal uniformities for the Instrument Line Shape characterization of the IRS instrument. 


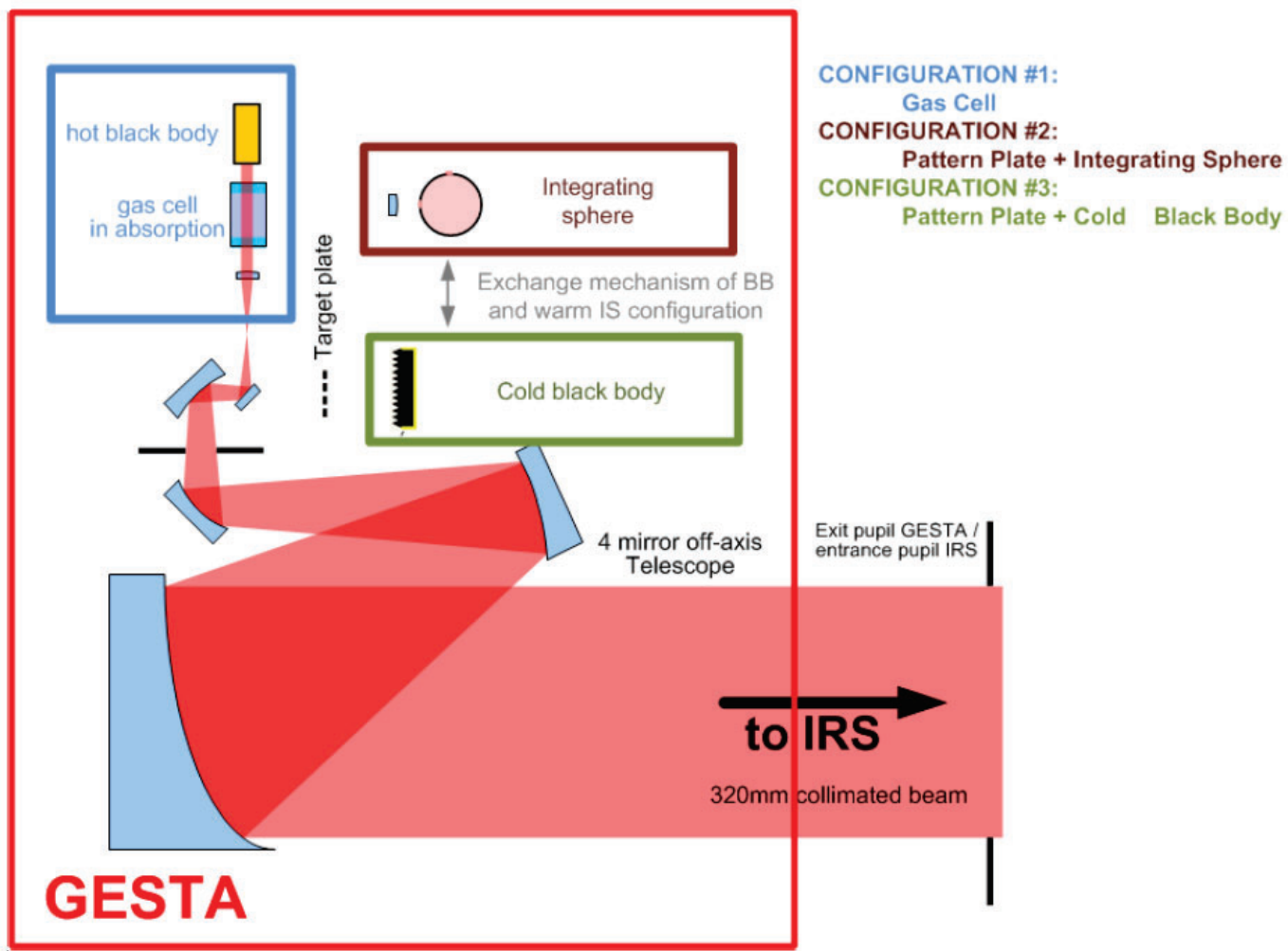

Figure 3. Schematic view of GESTA's lighting configurations

\subsection{MTG-IRS ILS characterization needs}

The Instrument Line Shape (ILS) corresponds to the shape of the response of the instrument to a monochromatic source. The main objectives of the ILS characterization on-ground are:

1) to estimate the difference between averaged measured ILS and ILS delivered by a mathematical model; and

2) to identify ghost residuals and shape instabilities. The accuracy of the retrieved ILS shape is required to be better than $4 \%$ of the actual ILS shape.
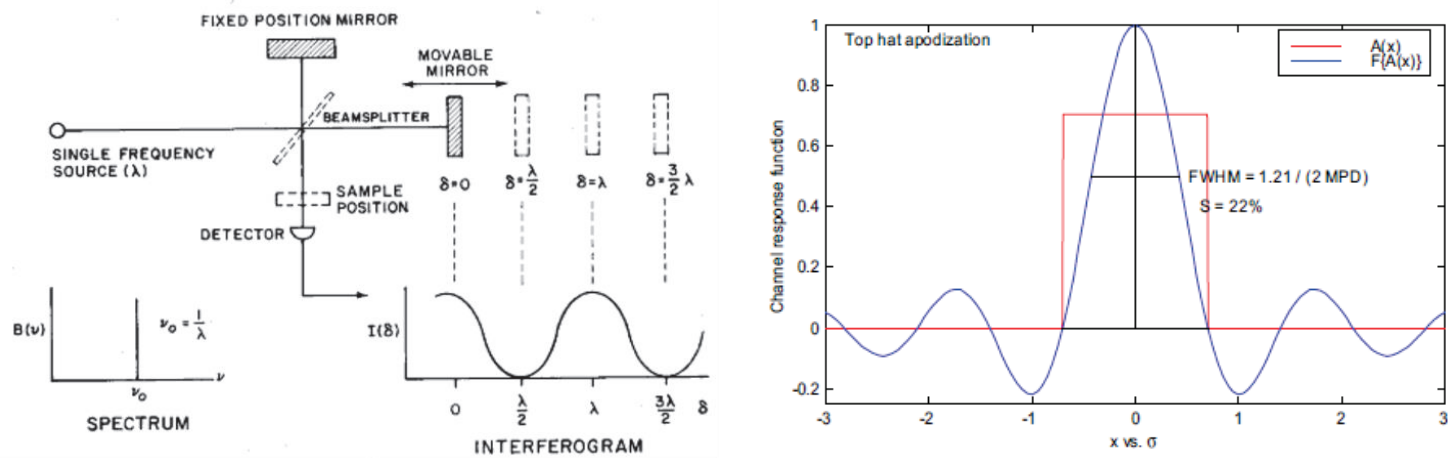

Figure 4. Instrument's acquisition principle (left) and typical Line Shape (right)

In order to estimate the ILS of the instrument in the two IR bands, a quasi-monochromatic spectral stimuli is thus required. Typically, absorption or emission features from well characterized gas cells can be employed. However, given the stringent contrast and SNR requirements for the on-ground characterization measurements, especially on the LWIR band, it is required to employ laser sources which provide higher spectral purity, contrast, and stability. The ILS characterization is performed using two different laser sources: 
- Carbon monoxide laser (CO) with primary peak at $5.43 \mu \mathrm{m}(1841.6 \mathrm{~cm}-1)$

- Carbon dioxide laser (CO2) with primary peak at $10.59 \mu \mathrm{m}$ or $(944.29 \mathrm{~cm}-1)$

These IR laser sources are injected in the integrating sphere to comply the FOV and pupil size requirements. The output port of the sphere is conjugated with the entrance pupil (PE plane) of the collimator illuminating MTG-IRS. A schematic view of the optical design between the sphere and this plane is given hereafter:

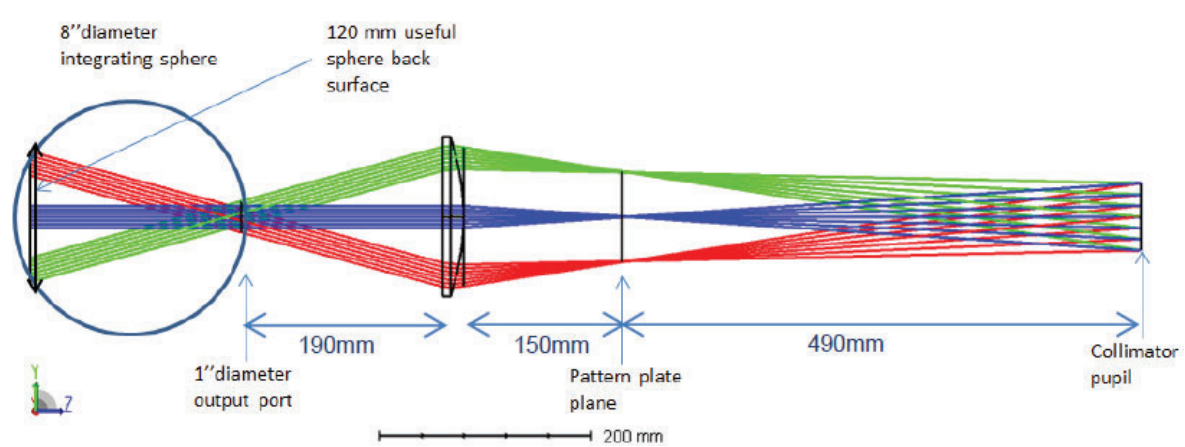

Figure 5. Optical design used to illuminate the entrance pupil of the collimator

By using these high-power and high spectral purity Infrared (IR) laser sources (with bandwidth $<200 \mathrm{MHz}$ ), coherent effects appear. At this level of the GESTA system, the IRS pupil and FoV uniformities specifications impact uniformities in the two planes seen on Figure 5, called PP plane (Pattern Plate plane), and PE plane (Pupil Entrance plane) which corresponds respectively to the entrance pupil of the collimator and the exit pupil of the GESTA.

In these two planes of interest, multiple diffuse reflections of the monochromatic laser radiation inside the integrating sphere produce a field at the exit aperture that is of random phase. The coherent light scattered from a diffuse surface produces a speckle pattern. This pattern is composed by an intensity varying from darkest, in the case where the sum of electromagnetic fields interferes destructively, to brightest if the interferences are constructive $[1,2]$.

To mitigate the effect of speckle and the associated degradation to the uniformity of illumination, a moving element is added on the laser beam path to do a dynamic variation of the electromagnetic fields summed at a given point, giving therefore temporal variations of the uniformity of the input radiation. Fast movements are necessary to average speckle patterns within the detector integration time $(\sim 400 \mu \mathrm{s})$, such that the associated white noise and the resulting ILS noise error are reduced. For the OGSE GESTA, the requirements of spatial uniformities are the following:

- The rms of the spatial variation of the radiance by the MWIR \& LWIR laser-sources over the exit pupil of the GESTA shall be less than $4.1 \%$,

- The rms of the spatial variation of the radiance by the MWIR \& LWIR laser-sources within its spectral bandwidth GESTA over the FOV shall be less than $15 \%$.

Moreover, as the modulation frequency of the moving element causes temporal variation, the requirement on temporal uniformities has been defined as follows:

- In case of a static target position, the rms of the temporal variation of the radiance by the MWIR laser-source at the exit pupil of the GESTA shall be smaller than $0.1 \%$, over 15 seconds, for temporal variations up to $150 \mathrm{~Hz}$

- In case of a static target position, the rms of the temporal variation of the radiance by the LWIR laser-source at the exit pupil of the GESTA shall be smaller than $0.1 \%$ over 15 seconds, for temporal variations up to $150 \mathrm{~Hz}$

Doing a modulation frequency to average the speckle patterns causes also another limitation: the intensity variation due to the speckle scrambler movement causes sidebands or spectral ghosts. When the spectral ghosts are close to the laser line of interest, they also distort the recorded ILS and lead to significant errors on the ILS shape and noise estimation. For example, the plot below on the left shows that the sideband generated for a modulation frequency of $166 \mathrm{~Hz}$ (caused by setting the motor speed at $10,000 \mathrm{rpm}$ ), overlaps almost directly with the LWIR laser line frequency of $165 \mathrm{~Hz}$ (resulting from multiplying the instrument OPD scanning speed of $0.175 \mathrm{~cm} / \mathrm{s}$ by the laser wavenumber of $944 \mathrm{~cm}-1$ ), so it is not a good option for the test. 


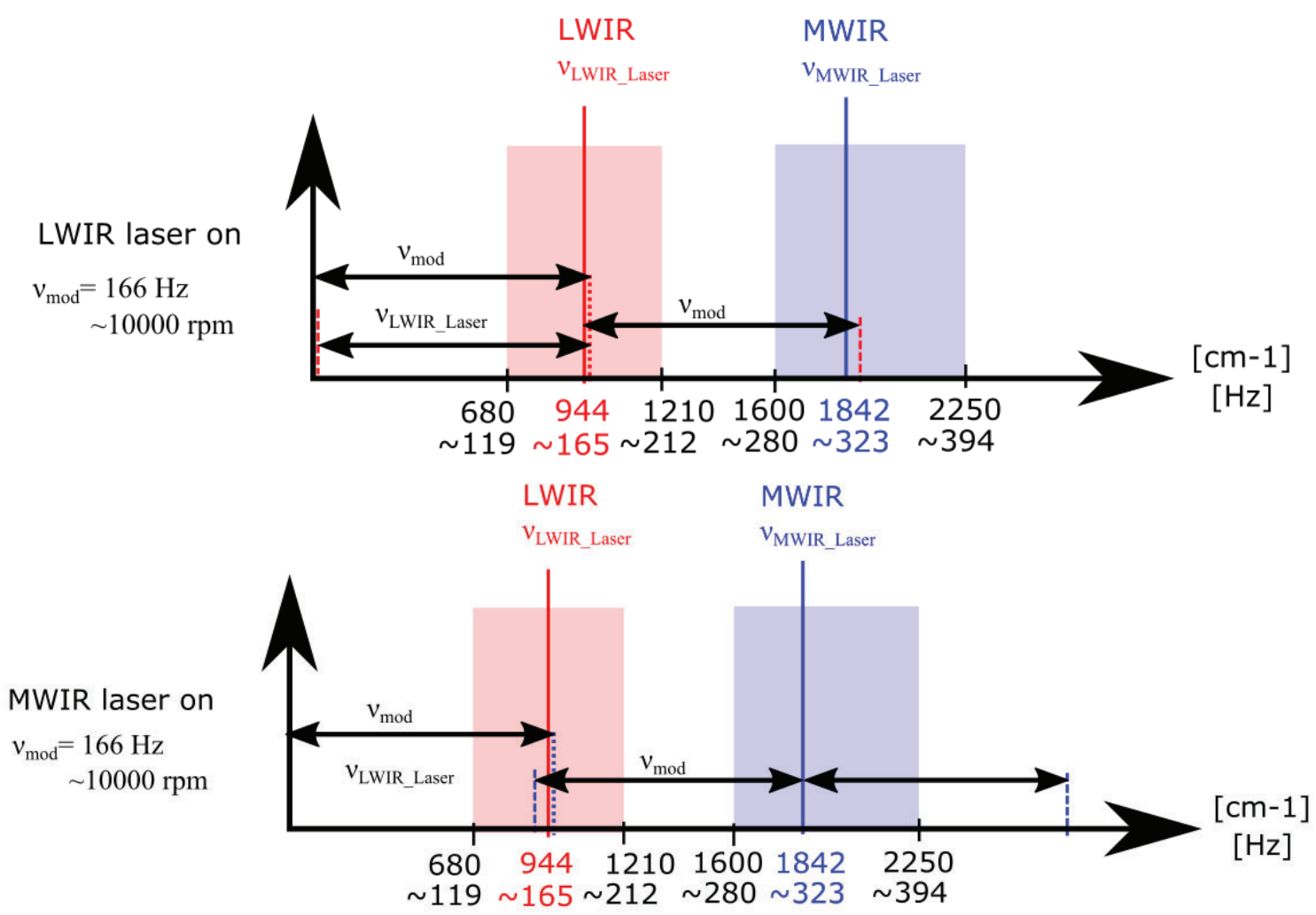

Figure 6. Representation of spectral ghosting due to the modulation frequency of the speckle scrambler

\section{SOLUTION DEVELOPED FOR SPATIAL UNIFORMITY OPTIMIZATION}

The sub part of illumination of the collimator by the OGSE GESTA is developed by Bertin Technologies, the associated optical study has evaluated, from a theoretical aspect, the radiance uniformity and stability achievable. The spatial nonuniformity is due to several contributors:

- The geometrical design, depending on the uniformity of the radiance at the output port of the sphere (design of the sphere), the uniformity of transmission and coatings on the IR lens, the optical quality of the image of the output port by the lens (design of the lens);

- The speckle pattern.

Each term has been evaluated and a budget allocated for each of them has been estimated. Thus, concerning the speckle aspects, the non-uniformity due to these coherent effects has to be less than $10 \%$ in the PP plane, and less than $3 \%$ in PE plane to comply the uniformity requirement of GESTA. This allocation shows the necessary mechanism to implement in order to reduce the non-uniformity due to the speckle.

Adding a dynamic variation on an element is a well-known method to average the speckle patterns [3-9]. Different speckle scrambler systems can be used. In the OGSE GESTA, to comply the environmental constrains (the OGSE is used in a vacuum chamber) and the uniformities requirements, Bertin Technologies has developed an innovative design by using a rotating diffusing disc inside the integrating sphere. The laser beams are inserted in the sphere, to hit the scrambler at a location giving the maximum of dynamic variation. The following figure gives a schematic view of the system: 


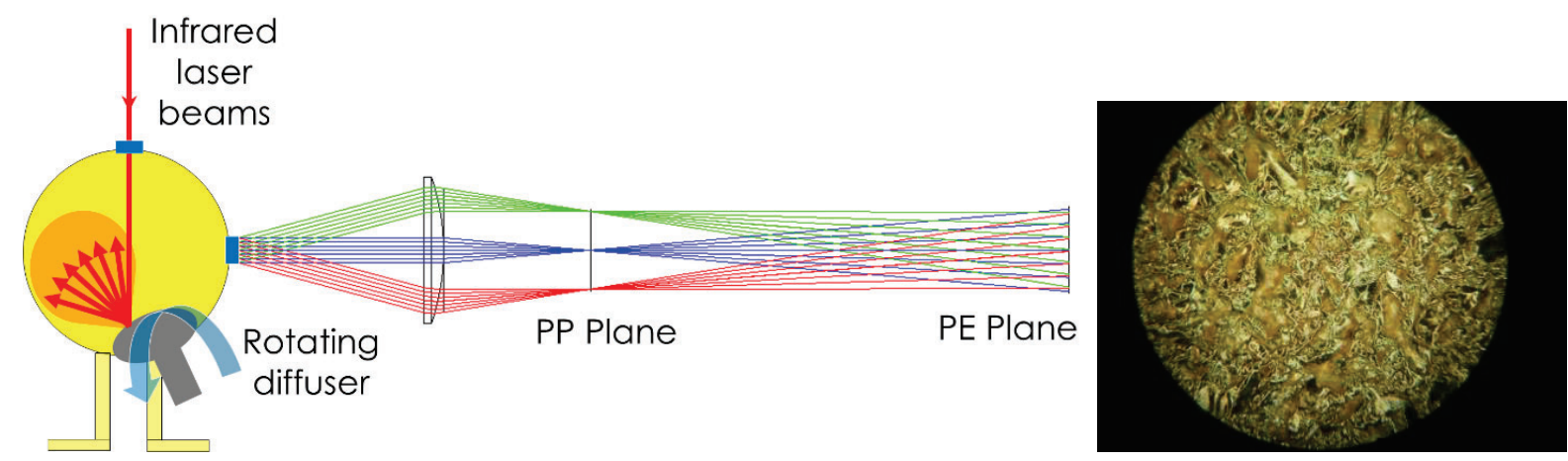

Figure 7. Basic principle of the speckle scrambling mechanism used in GESTA (on the left) and Infragold coating sample photography (on the right).

The speckle scrambler is located inside the sphere in order to get advantage of the multiple reflections given by this arrangement, enhancing the dynamic variation speed of the speckle pattern and thus its scrambling within detector integration time. The integrating sphere and the diffuser are manufactured in Infragold, the surface structure is given in Figure 7. A grain size of Infragold of $\sim 100 \mu \mathrm{m}$ is measured. To estimate the speckle scrambling efficiency, an intuitive simplest approach consists to suppose that a single incident ray hits only one time the moving Infragold surface during the integration time of the optical detector. Hence, in order to guarantee a speckle pattern complete change, the grains must cover a distance $\mathrm{L}$ higher than $\sim 100 \mu \mathrm{m}$. Taking a security factor of 2 , speckle scrambling optimisation is based on a minimum distance of $200 \mu \mathrm{m}$. This approach does not consider the possible multiple reflection of a ray on the rotating diffuser before going outside the sphere, and corresponds thus to a worst case.

With this approach, the speckle scrambling efficiency (in term of signal to noise ratio) increases with the square root of the number of grains seen by the laser spot during the integration time. A similar behaviour is expected with the rotation speed of the disc: the signal to noise ratio will have a square root dependence with the rotation speed of the motor. Consequently, if $\mathrm{D}$ corresponds to the distance between the laser spot on the rotating disc and its rotation axis, speckle scrambling efficiency increases as D increases. A minimum distance can be defined to get speckle scrambling for an optical ray, corresponding to the minimum distance for which two decorrelated speckle patterns are obtained.

To illustrate this, the Figure 8 gives the minimum distance $\mathrm{D}_{\min }$ between the incident ray on the diffusing surface and its rotation axis, as a function of the rotation speed $\mathrm{N}$, required to get speckle scrambling with a rotating Infragold diffusing surface. The integration time considered is $\tau_{\mathrm{i}}=400 \mu \mathrm{s}$ and $\tau_{\mathrm{i}}=100 \mu \mathrm{s}$ in this case.
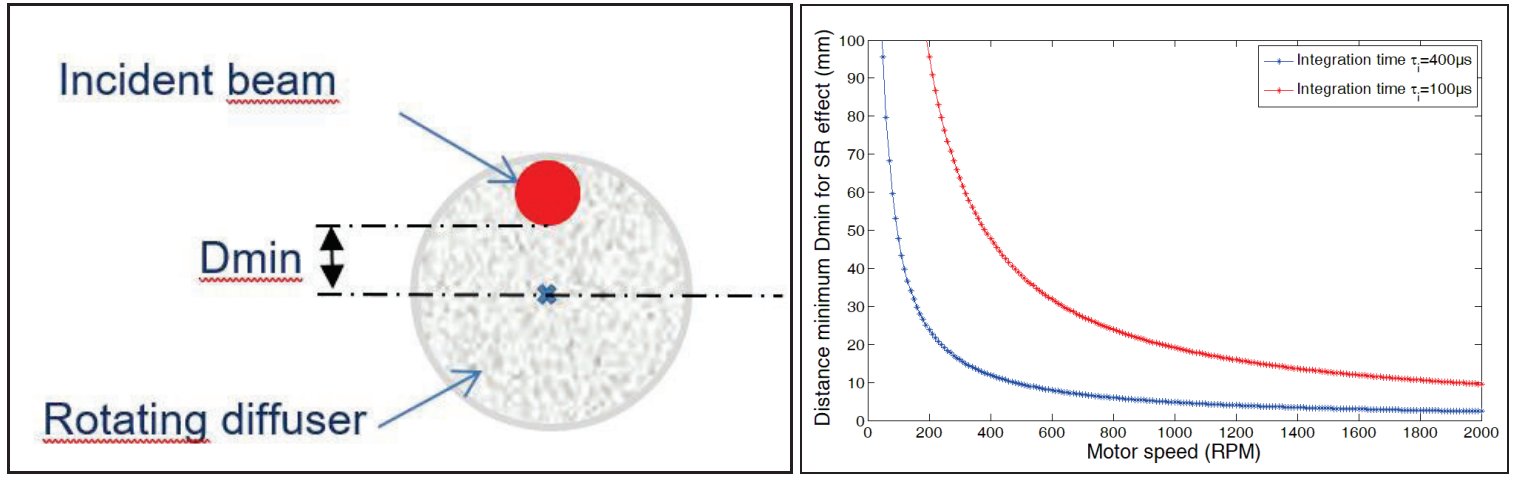

Figure 8. Variation of Dmin with the rotation speed $\mathrm{N}$ for a given set of physical parameters envisaged in GESTA (theoretical curve).

Thus, this innovating method of speckle scrambler gives the advantage to have two influencing parameters to maximize the efficiency: the distance between the laser spot on the disc and its rotation axis, and the motor speed. 


\section{BREADBOARD ACTIVITY}

Before assembling the mechanism of speckle scrambling in the OGSE, a breadboard activity has been conducted to evaluate the spatial uniformity in the two planes of interest, and measure the speckle scrambling efficiency. The activity consists in considering components as similar as possible as the ones integrated in the OGSE GESTA. To have access to the uniformity in the two planes of interest, a $\mathrm{LN}_{2}$-cooled infrared MCT detector is used, combined with a chopper device to produce the required modulation on the amplified signal. The MCT detector is also placed sequentially in the PE plane and the PP plane. Then, the uniformity is evaluated by sampling in these planes the pupil, consisting in measuring the signal intensity on a few points in the detector plane (displacement of the MCT detector in the considered pupil):

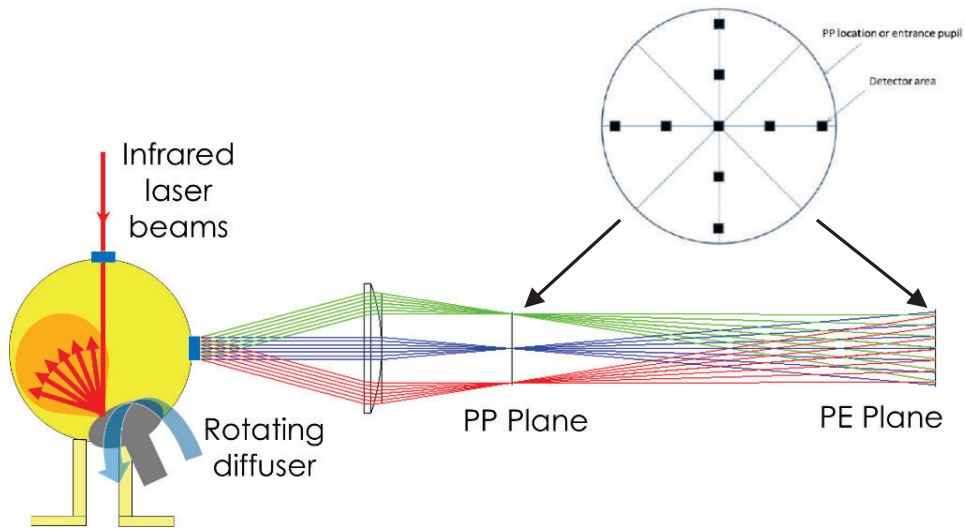

Figure 9. View of the sampling done by translating the detector in the plane of interest to measure the uniformity in the FOV and Pupil of GESTA

The breadboard activity focused on the influencing parameters, to achieve the best performances of spatial uniformities and temporal stability. The pictures given in Figure 10 show the set-up developed for this activity.

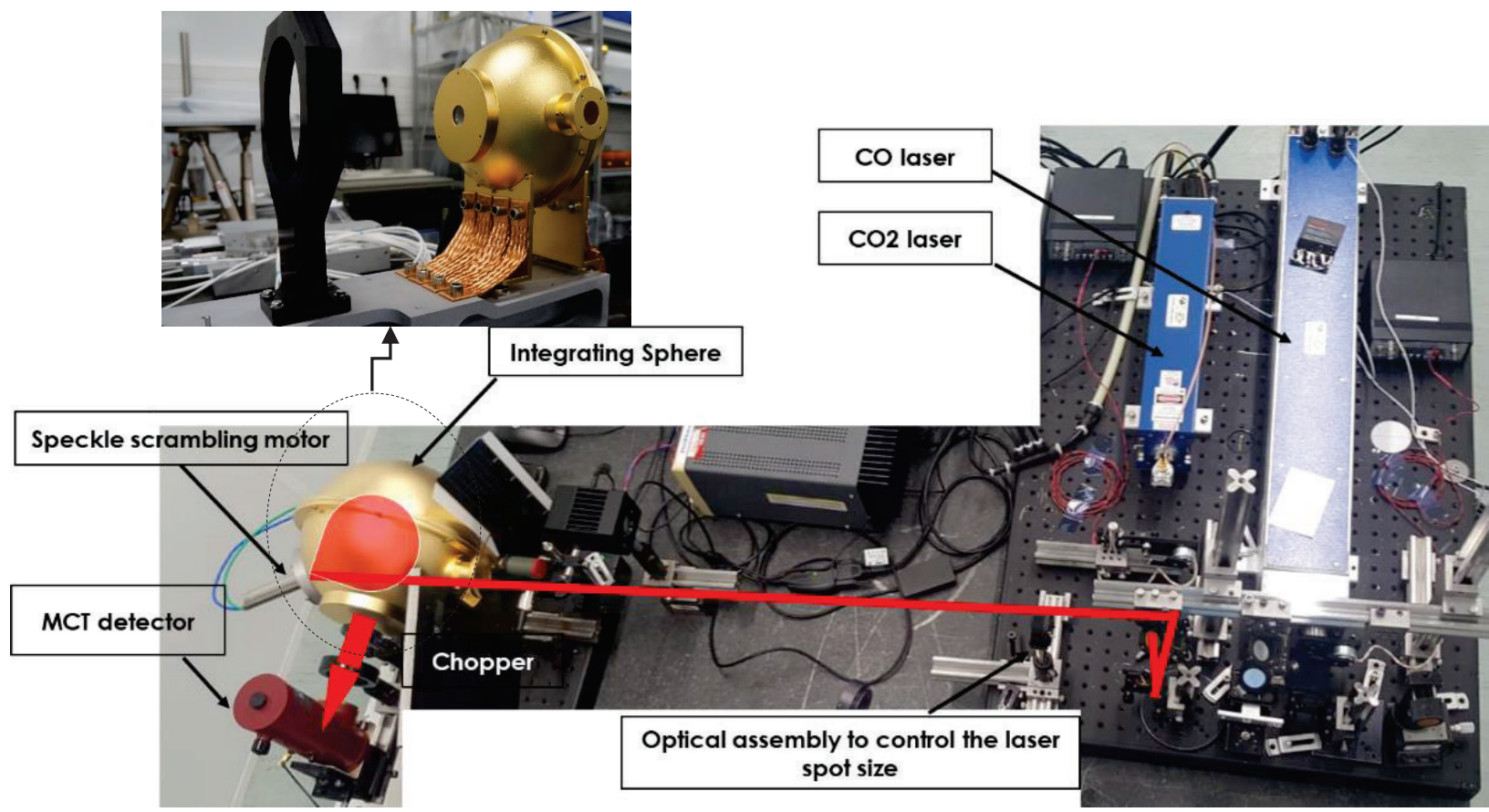

Figure 10. Picture of the integrating sphere in front of the IR lens (on the top), and complete set-up developed to measure the speckle scrambler efficiency (right). The IR laser beams are added in red lines. 
The geometrical physical parameters of the optical design (numerical aperture, output port diameter) are adjusted in order to obtain a similar etendue in the measurement than the IRS etendue.

Hereafter is a Zoom in on the sphere and the speckle scrambler motor support holding the diffuser inside the sphere:

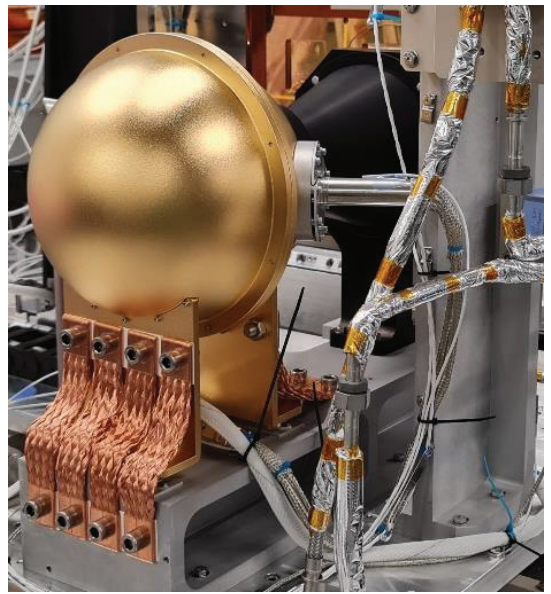

Figure 11. Picture of the integrating sphere with the speckle scrambler mechanism (on the left), zoom in on the rotating disc (right).

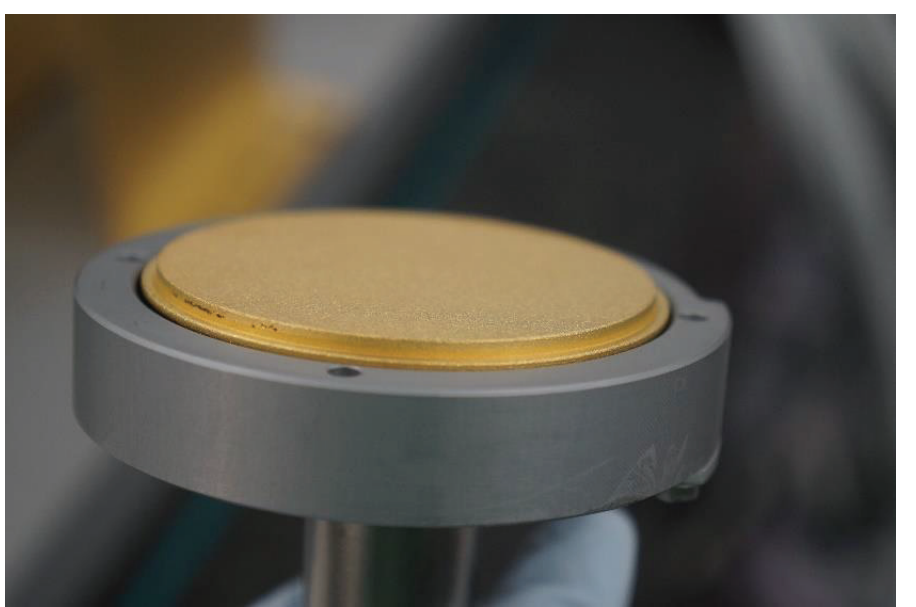

By doing the sampling in the pupil to compare the intensity between each location, in order to extract the uniformity, two supplementary contributors are implied in the comparison of signal intensity between each location:

- The stability of the laser sources;

- Others source of noises causing some variations in the radiance (shot noise mainly)

Thus, in order to be as accurate as possible, the laser sources are stabilized by using a water-cooled and stabilized circulation within typically $0.05^{\circ} \mathrm{C}$ of stability. For the $\mathrm{CO} 2$ laser, a piezo system is used in closed-loop to stabilize actively the output power. With this stabilization, the radiance variation due to the laser power variation is under $1 \%$.

The integrating sphere, the speckle scrambling mechanism and all components involved in the system of control have been developed to be vacuum compatible. For information, in the final OGSE GESTA, the laser sources are outside of the vacuum chamber and an optical window does the interface between the ambient and the interior of the chamber, as represented in Figure 2.

\section{RESULTS}

\subsection{Spatial uniformity}

The signal measured by the MCT detector, at a given location, is a succession of slots (the signal is chopped by placing a chopper on the laser emission path). The amplitude of each slot is relative to the radiance for this location. The evaluation of the uniformity, in a given plane, consists to extract, from the IR MCT detector signal, the mean value during the integration time $\tau_{\mathrm{i}}$ for all positions of the detector. The standard deviation is then extracted, to be confronted to the RMS variation specification on the radiance. 


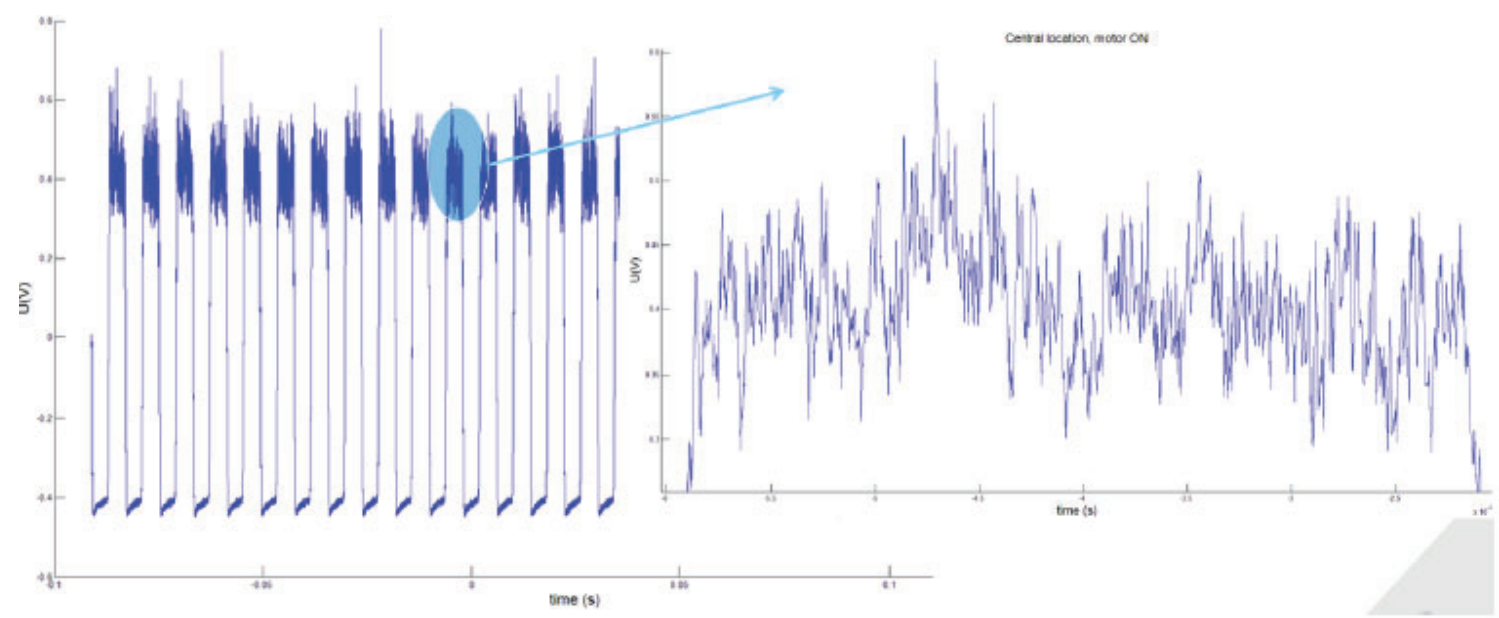

Figure 12. Example of signal measured as a function of time, here given for the central location of the detector.

The evaluation has been done for the values of $\tau_{\mathrm{i}}=400 \mu \mathrm{s}$ and $\tau_{\mathrm{i}}=100 \mu \mathrm{s}$. The following figure gives the result, for the requirement in the FOV of GESTA. The relative variation @ $1 \sigma$ is reported, for different rotation speeds. The experimental configuration is done with the $\mathrm{CO}_{2}$ laser source, for a $6.6 \mathrm{~mm}$ laser beam diameter on the disc. As expected, the uniformity increases with the motor speed, and shows a square root dependence with the speed.

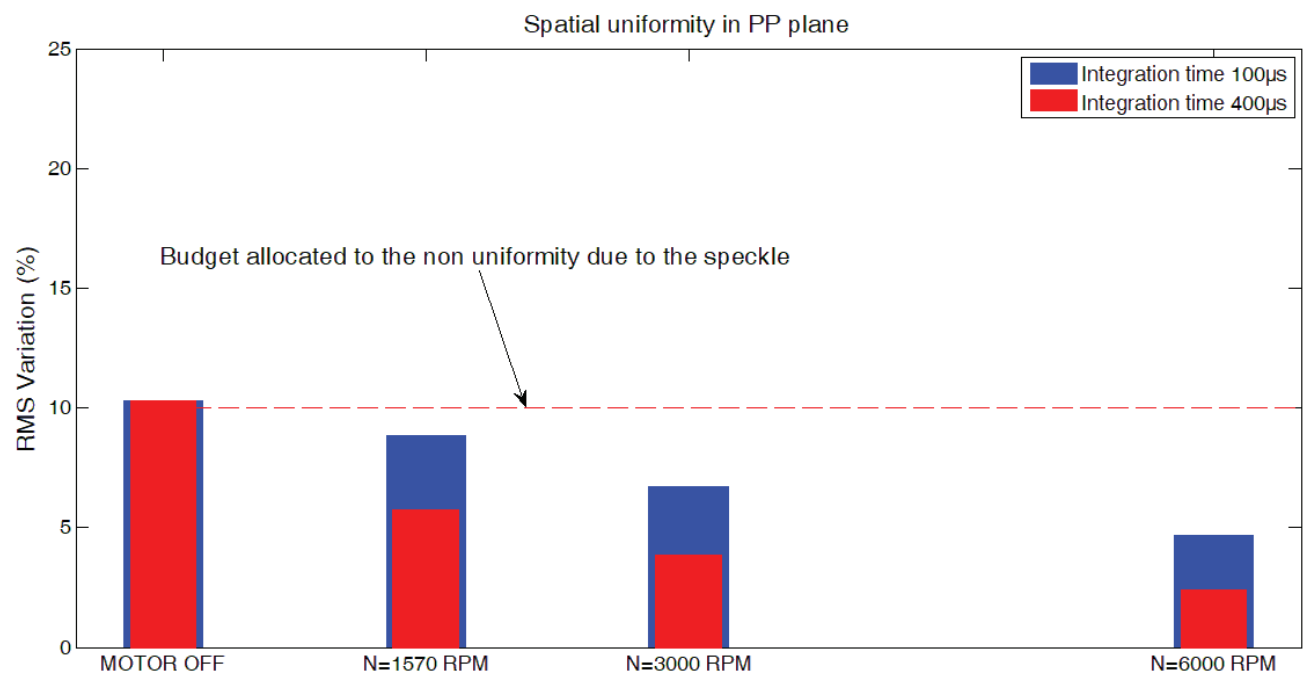

Figure 13. Spatial uniformity measured for several rotation speed. CO2 laser is used, with a $6.6 \mathrm{~mm}$ laser beam diameter on the disc.

When the rotation speed is zero, the RMS radiance variation is superior to $10 \%$. By increasing the rotation speed, the setup shows good enhancement, and lower value of $2.4 \%$ is reached, for a rotation speed of 6000 RPM.

The activity evaluated the radiance uniformity in the pupil (PE plane), with a similar signal process. A mechanical study gives a maximum rotation speed (around 3500 RPM) for which the vibrations caused by the speckle scrambler does not impact the measurement. The Table 1 summarizes the results obtained: 
Table 1. Summarize of the results obtained for different experimental configurations.

\begin{tabular}{|c|c|c|c|c|c|}
\hline \multirow{2}{*}{ Laser sources } & \multirow{2}{*}{$\begin{array}{l}\text { Speed N = } \\
\sim 3500 \text { RPM }\end{array}$} & \multicolumn{2}{|c|}{$\begin{array}{c}\text { Spatial uniformity in PP plane } \\
\text { (FOV of GESTA) }\end{array}$} & \multicolumn{2}{|c|}{$\begin{array}{l}\text { Spatial uniformity in PE plane } \\
\text { (exit pupil of GESTA) }\end{array}$} \\
\hline & & Measured & Requirement & Measured & Requirement \\
\hline \multirow{2}{*}{$\begin{array}{l}\mathrm{CO} \text { laser source } \\
(\lambda=5.3 \mu \mathrm{m})\end{array}$} & $\begin{array}{c}\text { laser beam } \\
\text { diameter } \\
6.5 \mathrm{~mm}\end{array}$ & $7.3 \%$ & \multirow{4}{*}{$15 \%$} & $3.9 \%$ & \multirow{4}{*}{$4 \%$} \\
\hline & $\begin{array}{c}\text { laser beam } \\
\text { diameter } \\
13.7 \mathrm{~mm}\end{array}$ & $3.5 \%$ & & $3.6 \%$ & \\
\hline \multirow{2}{*}{$\begin{array}{l}\mathrm{CO}_{2} \text { laser source } \\
(\lambda=10.59 \mu \mathrm{m})\end{array}$} & $\begin{array}{c}\text { laser beam } \\
\text { diameter } \\
6.6 \mathrm{~mm}\end{array}$ & $5.7 \%$ & & $5 \%$ & \\
\hline & $\begin{array}{l}\text { laser beam } \\
\text { diameter } \\
11 \mathrm{~mm}\end{array}$ & $3.8 \%$ & & $3.9 \%$ & \\
\hline
\end{tabular}

\subsection{Temporal stability}

As previously said, the uniformity has to be also checked in temporal stability considerations. In parallel to the experiments conducted for spatial uniformity measurements, the temporal variation has been analysed to evaluate the stability in time of the radiance, and the compliance to the requirement. The temporal instabilities of the radiance are mainly due, in this case:

- To the radiance variation caused by the rotation of the disc,

- The stability of the laser sources

- The others sources of noise (shot noise, thermo-mechanical stabilities, etc...)

This requirement applies to the case of a static target position. In other terms, it is considered here, the stability of the signal which will be seen by a pixel of IRS. The spatial uniformity is not involved, and it is possible to consider motor speed higher than the thresholds fixed for the spatial uniformity evaluation. We have evaluated the influencing parameters on this temporal stability. The following figure gives the result for the case $\mathrm{CO} 2$. The square root dependence with the motor speed is measured, as for the spatial uniformity measurement. For the dependence with the laser beam diameter on the rotating disc, the effect does not follow this law. The enhancement measurement is certainly due to an more important average of the area seen by the beam on the disc. 


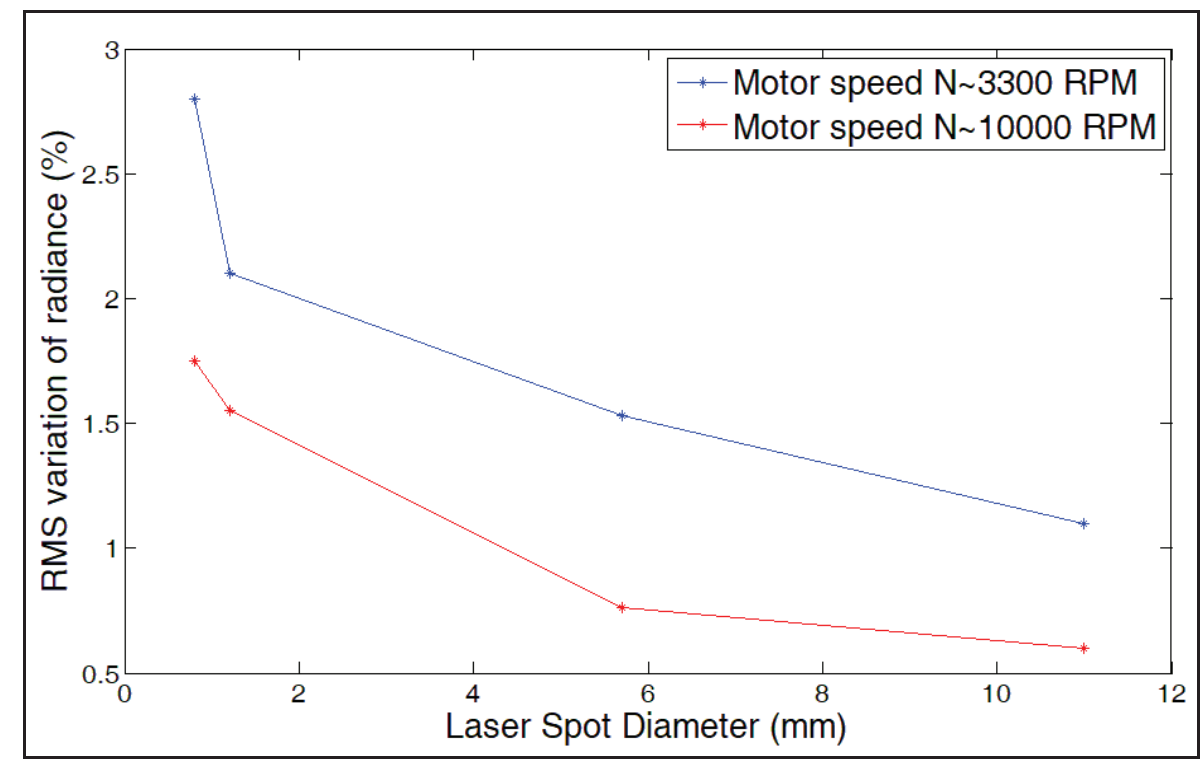

Figure 14. RMS variation of the radiance (in time, over 15s) with influencing parameters laser spot size and motor speed.

The measurement performed on this breadboard activity shows interesting results for high speed and high laser spot diameter value. These influencing parameters have a physical limitation:

- The physical limitations to the motor speed and the laser spot size on the disc have to be considered. Indeed, a limitation on the motor speed is given by the increase of the temperature of the motor, and the loss of voltage in the electrical cables due to the long distance inside the vacuum chamber.

- Concerning the laser spot size, a maximum value is considered to guarantee that the laser spot, with the optomechanical tolerances in the system, hits the disc.

Once these maximum acceptable values have been evaluated for the GESTA system, the temporal stability has been evaluated for the laser wavelengths used. The Table 2 summarizes the final configuration retained.

Table 2. Final result obtained on temporal stability of the radiance. The motor speed considered here is $10000 \mathrm{RPM}$.

\begin{tabular}{|c|c|c|c|}
\hline \multirow{2}{*}{$\begin{array}{c}\text { Laser sources } \\
\text { Laser spot size } \\
\text { value }(\mathrm{mm})\end{array}$} & \multicolumn{2}{|c|}{$\begin{array}{r}\text { Spatial uniformity in PE plane } \\
\text { (exit pupil of GESTA) }\end{array}$} \\
\cline { 3 - 4 } & & Measured & Requirement \\
\hline $\mathrm{CO}$ laser source $(\lambda=5.3 \mu \mathrm{m})$ & 13.7 & $\mathbf{0 . 3 \%}$ & $0.1 \%$ \\
\hline $\mathrm{CO}_{2}$ laser source $(\lambda=10.59 \mu \mathrm{m})$, & 11 & $\mathbf{0 . 6 \%}$ & \\
\hline
\end{tabular}

The results obtained shows a good enhancement on temporal stability, and the best achievable set of physical parameters. The final results can be accepted at this stage of development of GESTA. Further measurements will be carried out on the final configuration (GESTA completely assembled for test phases) to definitely demonstrate the compliance to the need concerning this aspect.

\section{CONCLUSION}

High resolved spectrometers embedded in MTG-IRS requires the use of OGSE to measure the spectral characterization and optical performances of the system, in the infrared spectral range. To comply the radiance level and stability, high stable and spectral purity infrared laser sources, associated to an integrating sphere, are used. To reach a radiance uniformity replying to the requirements and affording the possibility to measure the ILS with the required accuracy, 
Bertin Technologies associated to the sphere a mechanism of rotation disc. The breadboard activity conducted during the GESTA project has evaluated, in terms of spatial uniformity, and temporal stability, the benefits and the impact of this system.

A dedicated set-up, sensitive to the infrared radiance in the FOV and the exit pupil of the OGSE GESTA, has been developed to measure the radiance spatial uniformity and temporal stability. We have shown that this solution provides a high performing efficiency mean to average the speckle pattern, and shows a speckle reduction factor compliant to the need.

The solution proposed by Bertin Technologies provides a technological assembly compatible with severe environmental constrains (vacuum compatibility, temperature variation, vibrations), high radiance level achievement and usable in a high variety of spectral ranges.

The temporal and spatial speckle reduction performances presented in the previous sections will allow for a proper characterization of the IRS instrument ILS, even though they are declared partially compliant with respect to the initial GESTA requirements. Additional subsequent tests allowed to proof that the speckle scrambler rotation speed could be increased up to 12,200 rotations per minute $(\sim 203 \mathrm{~Hz})$. An increased rotation speed improves measurement accuracy by shifting the position of modulated ghosts far from the LWIR/MWIR lasers lines (cf Figure 6). Nevertheless, in order to have a correct interpretation of the ILS measurements at IRS level, additional measurements at different speckle scrambler rotation speeds will be carried out in order to identify and isolate the modulated ghost lines from the laser lines of interest.

This sub-system is proposed as an add-on for the OGSE developed by Bertin Technologies. Thus, Bertin Technologies has today the capability to provide and deliver fully multi-purposes optical systems, achieving highly performant illumination systems aiming at commissioning and calibrating complex systems as the MTG-IRS satellite can be.

\section{REFERENCES}

[1] J.W. Goodman, "Speckle phenomena in optics-theory and applications", Roberts and Company, USA, 2007.

[2] J. W. Goodman, in Laser Speckle and Related Phenomena, J. C. Dainty, ed. (Springer-Verlag, Berlin, 1984), pp. 9-75.

[3] S. Lowenthal and D. Joyeux, "Speckle removal by a slowly moving diffuser associated with a motionless diffuser", JOSA, 1971, 61(7), 847.

[4] C.S. Ih, "Speckle elimination using random spatial phase modulation", Applied Optics, 1977, 16(6), 1473.

[5] T. Stangner, H. Zhang, T. Dahlberg, K. Wiklund, and M. Andersson, "Step by step guide to reduce spatial coherence of laser light using a rotating ground glass diffuser", Applied Optics, 2017, 56(19), 5427.

[6] G.D. Boreman, Y. Sun and A.B. James, "Generation of laser speckle with an integrating sphere", Optical Engineering, 1990, 29(4), 339.

[7] J.I. Trisnadi, "Hadamard speckle contrast reduction", Optics Letters, 2004, Vol. 29, No. 1.

[8] S. Potvin and J. Genest, "Reducing the effect of integrating sphere speckle when characterizing the instrument line shape of a Fourier-transform hyperspectral imager", Applied Optics, 2009, 48(30)

[9] S. Kubota, and J.W. Goodman, "Very efficient speckle contrast reduction realized by moving diffuser device”, Applied Optics, 2010, 49(23) 